International Journal of Pure and Applied Mathematics

Volume 95 No. 4 2014, 593-596

ISSN: 1311-8080 (printed version); ISSN: 1314-3395 (on-line version)

url: http://www.ijpam.eu

doi: http://dx.doi.org/10.12732/ijpam.v95i4.12

ijpam.eu

\title{
AMNM PROPERTY ON VARIATION SEQUENCE SPACES
}

\author{
Leila Bagheri $^{1}$, Bahmann Yousefi $^{2} \S$ \\ ${ }^{1,2}$ Department of Mathematics \\ Payame Noor University \\ P.O. Box: 19395-3697, Tehran, IRAN
}

Abstract: In this paper, we will show that the spaces of p-bounded variation sequences are AMNM.

AMS Subject Classification: 47B37, 47A25

Key Words: bounded variation space, AMNM

\section{Introduction}

We write $\omega$ for the set of all complex sequences $x=\left(x_{k}\right)_{k=0}^{\infty}$. Let $\phi, l_{\infty}$ and $c_{0}$ denote the set of all finite, bounded and null sequences. We write $l_{p}=\{x \in \omega$ : $\left.\sum_{k=0}^{\infty}\left|x_{k}\right|^{p}<\infty\right\}$ for $1 \leq p<\infty$, and $b v=\left\{x \in \omega: \sum_{k=0}^{\infty}\left|x_{k}-x_{k-1}\right|<\infty\right\}$ for the set of all sequences of bounded variation and extend the definition to real $p \geq 1$ by putting $b v_{p}=\left\{x \in \omega: \sum_{k=0}^{\infty}\left|x_{k}-x_{k-1}\right|^{p}<\infty\right\}$ so that $b v_{1}=b v$. The set $b v_{p}$ also arise from the sets $l_{p}$, that is a sequence $x$ is in $b v_{p}$, if and only if the sequence $\left(x_{k}-x_{k-1}\right)_{k=0}^{\infty}$ is in $l_{p}$. It is this concept rather than the first one plays an important role in our studies.

By $e$ and $e^{(n)}\left(n \in N_{0}\right)$, we denote the sequences such that $e_{k}=1$ for $k=0,1,2, \ldots$ and $e_{n}^{(n)}=1$ and $e_{k}^{(n)}=0$ whenever $k \neq n$. For any sequence $x=\left(x_{k}\right)_{k=0}^{\infty}$, let $x^{[n]}=\sum_{k=0}^{n} x_{k} e^{(k)}$ be its n-section. A sequence $\left(b^{(n)}\right)_{n=0}^{\infty}$ in

Received: June 14, 2014

(C) 2014 Academic Publications, Ltd.

${ }^{\S}$ Correspondence author url: www.acadpubl.eu 
a linear metric space $X$ is called Schuader basis if, for every $x \in X$ there is a unique sequence $\left(\lambda_{n}\right)$ of scalars such that $x=\sum_{n=0}^{\infty} \lambda_{n} b^{(n)}$. An $F K$ space is a complete linear metric sequence space with the property that convergence implies coordinatewise convergence. A $B K$ space is a normed $F K$ space. An $F K$ space $X$ containing $\phi$ is said to have $A K$ if every sequence $x=\left(x_{k}\right)_{k=0}^{\infty} \in X$ has a unique representation $x=\sum_{k=0}^{\infty} x_{k} e^{(k)}$, that is $x=\lim _{n \rightarrow \infty} x^{[n]}$.

If $A$ is a Banach algebra, then the set of all linear functionals on $A$ is denoted by $A^{*}$ and the set of all its nonzero multiplicative functionals is denoted by $\hat{A}$. If $\varphi \in A^{*}$, then define $\check{\varphi}(a, b)=\varphi(a b)-\varphi(a) \varphi(b)$ for all $a, b \in A$. If $\delta \in \mathbb{R}^{+}$, we say that $\varphi$ is $\delta$-multiplicative, whenever $\|\check{\varphi}\| \leq \delta$. Also for each $\varphi \in A^{*}$ define $d(\varphi)=\inf \{\|\varphi-\psi\|: \psi \in \hat{A} \cup\{0\}\}$. Also, we say that $A$ is an algebra in which approximately multiplicative functionals are near multiplicative functionals, or $A$ is $A M N M$ for short, if for each $\varepsilon>0$ there is $\delta>0$ such that $d(\varphi)<\varepsilon$ whenever $\varphi$ is a $\delta$-multiplicative linear functional.

B. E. Johnson has shown that various Banach algebras are $A M N M$ and some of them fail to be $A M N M$ ([1]). Also, this property is still unknown for some Banach algebras such as $H^{\infty}$ and Douglas algebras. In this paper we will show that $b v_{p}$ is $A M N M$. For this topics on this sources see [1-4].

\section{Main Results}

In this section we investigate $b v_{p}$ as a Banach algebra that is $A M N M$. It has been proved that $b v_{p}$ is a Banach space with $B K$ property.

Lemma 2.1. (i) If $x=\left(x_{k}\right)_{k=0}^{\infty} \in b v_{p}$, then $x$ is a bounded sequence.

(ii) If $x=\left(x_{k}\right)_{k=0}^{\infty}, y=\left(y_{k}\right)_{k=0}^{\infty} \in b v_{p}$, then $x y=\left(x_{k} y_{k}\right)_{k=0}^{\infty} \in b v_{p}$.

(iii) The space $b v_{p}$ is a commutative Banach algebra.

Proof. (i) Since $x_{k}=x_{0}+x_{1}-x_{0}+x_{2}-x_{1}+\ldots+x_{k}-x_{k-1}$ for all integers $k \geq 0$, we get

$$
\begin{aligned}
\left|x_{k}\right|^{p} & =\left|x_{0}+\left(x_{1}-x_{0}\right)+\left(x_{2}-x_{1}\right)+\ldots+\left(x_{k}-x_{k-1}\right)\right|^{p} \\
& \leq 2^{p} \sum_{k=0}^{\infty}\left|x_{k}-x_{k-1}\right|^{p}=2^{p}\|x\|_{b v_{p}}^{p},
\end{aligned}
$$

Thus $\sup _{k}\left|x_{k}\right|<\infty$. 
(ii) Note that

$$
\begin{aligned}
\sum_{k=0}^{\infty}\left|x_{k} y_{k}-x_{k-1} y_{k-1}\right|^{p} & =\sum_{k=0}^{\infty}\left|x_{k}\left(y_{k}-y_{k-1}\right)+y_{k-1}\left(x_{k}-x_{k-1}\right)\right|^{p} \\
& \leq\left\{\|x\|_{\infty}\|y\|_{b v_{p}}+\|y\|_{\infty}\|x\|_{b v_{p}}\right\}^{p}
\end{aligned}
$$

So by part (i) it follows that $x y \in b v_{p}$.

(iii) Since $b v_{p}$ is a $B K$ space and convergence implies coordinate wise convergence, thus the right multiplications $x \mapsto y x$ and the left multiplications $x \mapsto x y$ are continuous. Now, clearly $\|x y\|_{b v_{p}} \leq\|x\|_{b v_{p}}\|y\|_{b v_{p}}$.

Lemma 2.2. (see [4]) Define the sequence $b^{(k)}=\left\{b_{n}^{(k)}\right\}_{k \in \mathbb{N}}$ of the elements of the space $b v_{p}$, by $b_{n}^{(k)}=1$ for all $n \geq k$ and otherwise 0 . Hence the sequence $\left\{b^{(k)}\right\}_{k \in \mathbb{N}}$ is a basis for the space $b v_{p}$ and any $x \in b v_{p}$ has a unique representation of the form $x=\sum_{k} \lambda_{k} b^{(k)}$, where $\lambda_{k}=x_{k}-x_{k-1}$ for all $k \in \mathbb{N}$.

Definition 2.3. Let $1<q<\infty$. Define the space $d_{q}$ consisting of all sequences $a=\left(a_{k}\right)_{k=0}^{\infty}$ normed by $\|a\|_{d_{q}}=\left(\sum_{k}\left|\sum_{j=k}^{\infty} a_{j}\right|^{q}\right)^{\frac{1}{q}}<\infty$.

Theorem 2.4. The space $b v_{p}^{*}$ is isometrically isomorphic to $d_{q}$.

Proof. Define the transformation $A: b v_{p}^{*} \rightarrow d_{q}$ by $f \mapsto A f=\left(f_{0}, f_{1}, f_{2}, \ldots\right)$ where $f_{j}=f\left(e^{(j)}\right)$ for each $j \in \mathbb{N}$. Therefore we obtain that $\|f\|=\left(\sum_{k=0}^{\infty}\left|g_{k}\right|^{q}\right)^{\frac{1}{q}}$ where $g_{k}=\sum_{j=k}^{\infty} f_{j}$ for each $k \in \mathbb{N}$. Hence $A$ is an isometric isomorphism.

Theorem 2.5. If $1<p<\infty$, then $b v_{p}$ is $A M N M$.

Proof. Let $1<p<\infty$ and $\varepsilon \in(0,1)$, set $\delta=\frac{\varepsilon^{2}}{16}$ and consider $f \in b v_{p} *=d_{q}$ with $\|\check{f}\|<\delta$. If $\|f\| \leq \varepsilon$, then $d(f) \leq \varepsilon$. Therefore, suppose that $\|f\|>\varepsilon$. For each subset $E$ of $\mathbb{N}_{0}$, let $\alpha(E)=\left[\sum_{k \in E}\left|\sum_{j=k}^{\infty} f_{j}\right|^{q}\right]^{\frac{1}{q}}$. For any subsets $E$ and $F$ of $\mathbb{N}_{0}$, if $E \cap F=\phi$, then either $\alpha(E) \leq \frac{\varepsilon}{4}$ or $\alpha(F) \leq \frac{\varepsilon}{4}$. So if $E \subseteq \mathbb{N}_{0}$, we have that either $\alpha(E) \leq \frac{\varepsilon}{4}$ or $\alpha(E) \geq \frac{3 \varepsilon}{4}$. Note that, if $E, F \subseteq \mathbb{N}_{0}$ with $\alpha(E) \leq \frac{\varepsilon}{4}$ and $\alpha(F) \leq \frac{\varepsilon}{4}$, then $\alpha(E \bigcup F) \leq \frac{\varepsilon}{4}$. Since $\|f\|>\varepsilon$, there exists a positive integer $m$ such that $\alpha(m) \geq \frac{3 \varepsilon}{4}$, then $\alpha(\mathbb{N}) \leq \frac{\varepsilon}{4}$. Let $b^{(m)}$ be the $m$-th element of a shuader basis, $\left(b^{(k)}\right)_{k=0}^{\infty}$. We have $\left|\check{f}\left(b^{(m)}, b^{(m)}\right)\right|=\mid f\left(b^{(m)} \cdot b^{(m)}\right)-$ $f\left(b^{(m)}\right) \cdot f\left(b^{(m)}\right)|=| f\left(b^{(m)}\right)-f\left(b^{(m)}\right)^{2}|=| g_{m}-g_{m}^{2} \mid$, where $g_{m}=\sum_{j=m}^{\infty} f_{j}$. Since $\|\check{f}\|<\delta$, we get $\left|g_{m}-g_{m}^{2}\right|<\delta$. So either $\left|g_{m}\right| \leq 2 \delta$ or $\left|1-g_{m}\right| \leq 2 \delta$. But $\alpha(m)=\left|g_{m}\right| \geq \frac{3 \varepsilon}{4}$, thus $\left|1-g_{m}\right| \leq 2 \delta$. Now if $\psi$ is the evaluation functional defined by $\psi(a)=a_{m}$, then $\|f-\psi\|_{b v_{p}^{*}}=\left[\alpha(\mathbb{N})^{q}+\left|g_{m}-1\right|^{q}\right]^{\frac{1}{q}} \leq \frac{\varepsilon}{4}+2 \delta<\varepsilon$. Thus, indeed $d(f)<\varepsilon$ and the proof is complete. 


\section{References}

[1] B. E. Johnson, Approximately multiplicative functionals, Journal of the Landon Mathematical Society, 34, No. 3 (1986), 489-510.

[2] B. Aupetit, A Primer on Spectral Theory, Springer-Verlag, NewYork (1991).

[3] L. Bagheri and B. Yousefi, Reflexivity of the shift operator on some BK spaces, Rendiconti Del Circolo Matematico Di Palermo, 2013, DOI 10.1007/s12215-013-0143-5.

[4] A. M. Akhmedov and F. Basar, The fine spectra of the difference operator $\triangle$ over the sequence space $b v_{p}$, Acta Mathematica Sinica, 23, No. 10 (2007), 1757-1768. 